\title{
Description of the first instar larva of Euzonitis rubida with remarks on the systematics of the subfamily Nemognathinae (Coleoptera: Meloidae)
}

\author{
Andrea Di Giulio \& Marco A. Bologna*
}

\begin{abstract}
Di Giulio, A. \& Bologna, M. A. 2007: Description of the first instar larva of Euzonitis rubida with remarks on the systematics of the subfamily Nemognathinae (Coleoptera: Meloidae). — Entomol. Fennica 18: 102-109.

The first instar larva (triungulin) of Euzonitis rubida (Ménétriés, 1832) is described and illustrated. This is the first description of a triungulin of the nemognathine genus Euzonitis Semenow, 1893. This larva is very similar to that of the genus Zonitis Fabricius, 1775, the distinction of these two genera remaining difficult by using both larval and adult features. The examination at the scanning electron microscope of several characters of these and other genera of the subfamily Nemognathinae, confirms the main distinction of tribes, but better outlines the condition of some features previously incorrectly defined. Additional species of some genera were also examined and partially figured.
\end{abstract}

\begin{abstract}
A. Di Giulio \& M. A. Bologna: Dipartimento di Biologia, Università degli Studi "Roma Tre", Viale Marconi 446, 00146 Roma, Italy; * Corresponding author's e-mail: bologna@bio.uniroma3.it
\end{abstract}

Received 26 January 2006, accepted 21 August 2006

\section{Introduction}

The genus Euzonitis (Meloidae, Nemognathini) was erected by Semenov (1893) to include those species of Palaearctic Zonitis with an elongate, enlarged and spatulate outer hind tibial spur. Euzonitis now includes 18 species, some of doubtful validity (Bologna \& Pinto 2002), distributed in the Madeira Archipelago, the Mediterranean area, and in Western and Central Asia from Anatolia east to Mongolia. Though Borchmann (1942) also tentatively assigned the Namibian E. maculicollis to Euzonitis, this African species belongs to the genus Zonitis Fabricius, 1775 (Kaszab 1954, Bologna 2000, Bologna \& Pinto 2002).

There is little information on the biology of Euzonitis. Members of the genus are termophilous or xerophilous, living in sub-arid envi- ronments with steppe or Mediterranean vegetation. Adults are polyphagous, feeding especially on Asteraceae, but also on Apiaceae, Brassicaceae, Euphorbiaceae, Leguminosae and Lamiaceae. Incomplete field observations of the sexual behaviour of E. rubida (Ménétriés, 1832) in Turkey (Bologna 1991) indicates that, as in other Nemognathini, a simple brief dorsal activity occurs within courtship sequences. The only larval host information is for E. quadrimaculata (Pallas 1782) in Madeira recorded as a parasitoid on bees of the genus Anthophora Latreille, 1803 (Hymenoptera, Apoidea, Anthophoridae) (Lindberg 1963).

Though several authors have hypothesized the close relationship of Euzonitis and Zonitis based on adult morphology, the systematic position and the validity of the genus remained a matter of debate especially since its larvae remained 
unknown. Escherich (1897) considered Euzonitis as a subgenus of Zonitis, while Kaszab (1969), Bologna (1991), and Bologna and Pinto (2001) supported the original distinction proposed by Semenov (1893). The problem is that Zonitis itself is inadequately defined (Pinto \& Bologna 1999, Bologna \& Pinto 2001, 2002) and probably represents a polyphyletic group. The metatibial character of Euzonitis is an obvious autapomorphy but other features (male genitalia, mouthparts, antennae, etc.) clearly relate it to the "Zonitis complex". Differences among the described subgenera or recognised species groups of Zonitis (see Kaszab 1954, Enns 1956, Pinto \& Bologna 1999, Bologna \& Pinto 2002) include characters as relevant as those distinguishing $E u$ zonitis.

Recently we obtained the first instar larva, or triungulin, of E. rubida from southern Anatolia. Characters of this larva were utilised in a recent phylogenetic analysis of the family (Bologna \& Pinto 2001), but the triungulin remained undescribed.

The aim of this paper is to describe and illustrate the first instar larva of E. rubida. Larval morphology of this species, assumed to be representative of the genus, is compared to that of other genera of the tribe Nemognathini, contributing to the debate on the validity and phylogenetic position of the genus Euzonitis, in the perspective of the revision of the "Zonitis complex". Additionally, a S.E.M. analysis of first instar larvae of several nemognathine species is here reported to outline the variability of some characters among the genera, and improve the phylogenetic reconstruction of the subfamily Nemognathinae.

\section{Material and methods}

The description of the first instar larva of Euzonitis rubida is based on several (about 300) triungulins, hatched from eggs collected in the field, and then reared in the laboratory. Eggs were laid by a single female on a Carduus sp. in Turkey (Vilayet Içel, near Mut, 7.v.1991, M. A. Bologna). Triungulins hatched on 14.v.1991. Larvae are preserved in the M. A. Bologna Collection, Università "Roma Tre" (Roma, Italy).

First instar larvae are kept in 70\% ethanol (vi- als 119, 120, 125). Five triungulins were mounted on slides in Canada Balsam (M 125-129) and examined with Leitz Laborlux S microscope. Several triungulins, mounted on stubs $(63,115)$ after critical point dehydration and gold sputtering, were analysed and photographed using a scanning electron microscope (S.E.M.) Philips XL 30. Triungulins of species of other Nemognathinae genera were used for comparison (see Bologna \& Pinto 2001 for the list of examined material).

The S.E.M. examination of some triungulins of other Nemoganthinae used for our comparative analysis with E. rubida, pointed to the erroneous interpretation of certain features examined with the light microscope for a previous phylogenetic study (Bologna \& Pinto 2001). The first instar larvae of the following species were examined, some of which have not been described earlier: (a) Tribe Stenoderini: Stenodera puncticollis (Laporte de Castelnau, 1840), S. caucasica (Ménétriés, 1832); (b) tribe Horiini: Synhoria testacea (Fabricius, 1781); (c) tribe Nemognathini: Stenoria analis Schaum, 1859, Sitaris capensis Péringuey, 1886 (both previously considered Sitarini), Zonitis bellieri Reiche, 1860, Z. flava Fabricius, 1775, Z. immaculata (Olivier, 1775), Gnathium francilloni (Kirby, 1818), Nemognatha vansoni Kaszab, 1951, N. lutea LeConte, 1853, N. lurida (LeConte, 1853).

Terminology of larval features follows Parker and Böving (1924), MacSwain (1956), Lawrence (1991), and Bologna and Pinto (2001). For certain characters of larval chaetotaxy we adopted the notational conventions of Selander (1990), and Bologna and Di Giulio (2002). Measurements of first instar larvae reported in the description represent ranges from 5 slide-mounted larvae and were taken using a light microscope.

\section{Results}

\subsection{Description of first instar larva of Euzonitis rubida}

Habitus and microsculpture. Triungulin navicular in shape typically adapted to phoresy, short, about 3.4 times as long as greatest width (on mesothorax), rounded anteriorly, evenly narrowed posteriorly. Head, legs, terga and sterna 

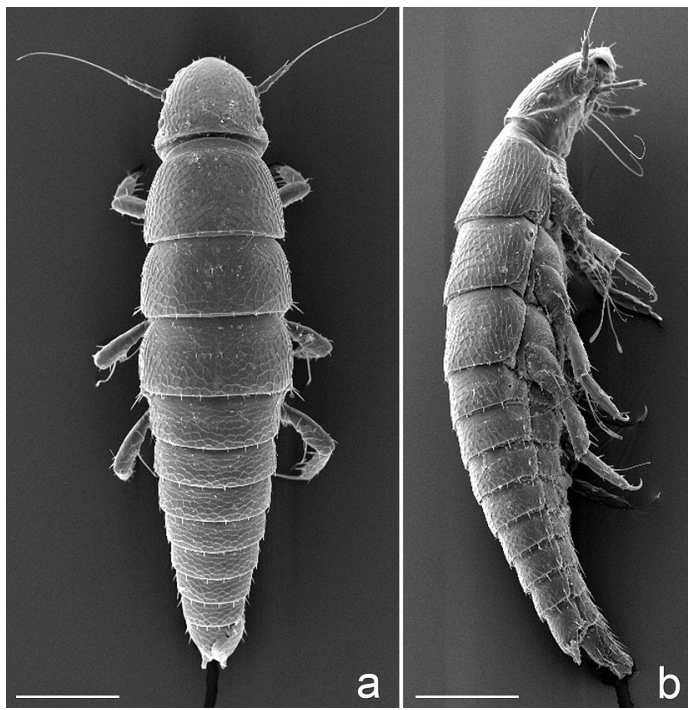

well sclerotised, with most setae short. Colour light-brown. Cuticle of sclerotised areas of the body reticulate with polygonal meshes, sub-hexagonal or sub-pentagonal on head and terga (Figs. 1a-1b, 2a, 2c, 2g, 2h), more elongate on legs, subtriangular, scale-like and imbricate on sterna; posterior meshes of sterna and laterotergites with elongate, spine-like prolongations posteriorly directed, giving a microserrate aspect to the posterior margins.

Dimensions. Body length (BL) 0,74-0,76 $\mathrm{mm}$ (from abdominal apex to clypeolabral suture); head length (HL) $0.15-0.16 \mathrm{~mm}$ (from occipital foramen to clypeolabral suture), maximum width (HW) $0.13-0.15 \mathrm{~mm}$; diameter of dorsal stemma (STD) 0.012-0.014 mm; antennal length (AL) $0.05 \mathrm{~mm}$, terminal antennal seta length (ASL) $0.15-0.17 \mathrm{~mm}$; prothorax length (PL) $0.1-$ $0.11 \mathrm{~mm}$, maximum width, at base (PW) 0.19-0.2 $\mathrm{mm}$; length of abdomen (ABL) $0.34-0.37 \mathrm{~mm}$, maximum width (ABW, at level of segment I) 0.17-0.19 mm; diameter of spiracles (SPD, external diameter of peritreme): mesothoracic $14 \mu \mathrm{m}$, abdominal I $8 \mu \mathrm{m}$.

Head (Figs. 2a-2c). Cephalic capsule broadly subtriangular, widest near base, behind stemmata; dorsal surface regularly convex, with anterior margin rounded (in dorsal view), and with transverse, straight, basal elevation posterior to stemmata. Cephalic capsule abruptly narrowing behind basal elevation forming a neck. Epicranial suture (stem + frontal arms) absent.

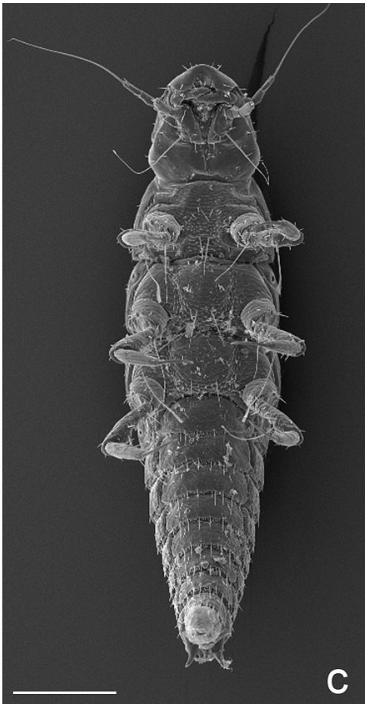

Fig. 1. First instar larva of Euzonitis rubida -a. Habitus, dorsal view $-b$. Habitus, right lateral view - c. Habitus, ventral view. Scale bar $=100 \mu \mathrm{m}$.

Two stemmata present on each side (Figs. 2a, 2c), distinctly convex, obliquely disposed, the dorsal one much larger (about double) and slightly posterior to the ventral one, and about as large as mesothoracic spiracle. Frons, clypeus and labrum fused without apparent sutures. Clypeolabral region (Figs. 2b-2c) greatly sloping, with medial emargination fitting the host seta; frontoclypeal row of setae (FCR) with three pairs of setae of about equal size, the lateral (FCR3) nearly ventral because of the folding of anterolateral frontoclypeus; one sensory pit near FCR3; four pairs of frontal setae of about equal size posterior to FCR: one lateral, slightly anterior to the antennal insertion, three longitudinally lined on frontal area, one large pair of pits present close to medial pair of frons. Each epicranial plate with five short setae (including ocular seta) around each pair of stemmata (Figs. 2a, 2c); ocular seta unmodified, subequal to the others, placed anterior to stemmata and to ocular sensory pit; ocular sensory pit slightly anterior to stemmata; three pair of setae transversally lined just anterior to basal elevation, one pair of large pits displaced anteriorly to medial pair; three minute setae and one small pit dorsally posterior to basal elevation. Labrum fused to frontoclypeus, some labral setae and pits present medioventrally; epipharynx with a dense tuft of thick setae, appearing under the light microscope as a tangled assemblage; basal margin of epipharynx completely sclerotised and closed. Mandibles ventral in position, short, falcate (Figs. 
Fig. 2. First instar larva of Euzonitis rubida

-a. Head, dorsal view

- b. Head, ventral view

-c. Head, right lateral

view - d. Right maxilla, ventral view - e. Right antenna and maxillary palpus, lateral view $-f$. Apex of left fore leg, anteroventral view - g. Abdominal terga III, right lateral view $-\mathrm{h}$. Apex of abdomen (segments VIII-IX), dorsal view. Scale bars: 50 $\mu \mathrm{m}$ (Figs. a-c); $20 \mu \mathrm{m}$ (Figs. d, f-h); $10 \mu \mathrm{m}$ (Fig. e).

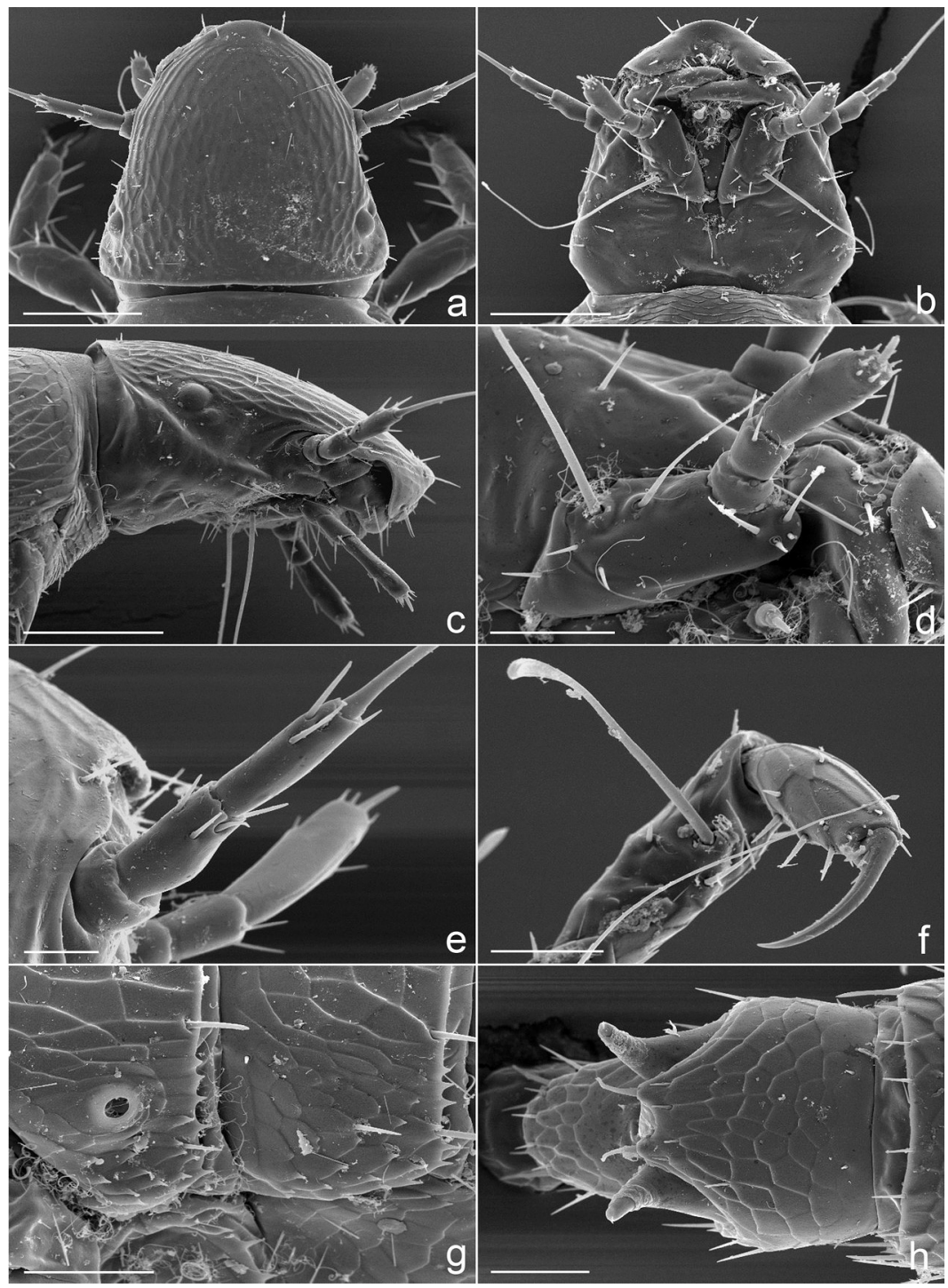

$2 \mathrm{~b}, 2 \mathrm{~d})$, moving in an obliquely vertical plane; base of mandibles broad, subrectangular, with a large ventral condyle; ental surface with three distinct, toothlike, transverse ridges, basal ridge weakly developed; lateral margin with two basal setae, one large pit mesodorsally. Maxillae (Fig. $2 d)$ with stipes short with two rows of setae: apical row (first row) with two setae, lateral seta normal, medial seta extremely long, about as long as prothorax, and slightly enlarged at apex; basal row (second row) with two short setae and one lateral pit; cardo absent; cardinal seta present; mala simple, lobiform, with five-six moderately long spiniform apical setae; maxillary palpi long, three-segmented, with segments I and II short, and segment III longer than I and II combined; segment II slightly longer than I, segment III three times longer than II, terminating with a small apical sensory area bearing a crown of seven-nine conical papillae and a medial conspicuous stick-like sensory appendix almost twice the length of others; segment I with one ventral pit; segment II with two ventrolateral subequal setae (one lateral and one medial); segment III with two medial setae and one slender, elongate, lateral digitiform sensillum. Antennae (Fig. 2e) about one-third as long as head, posteriorly or laterally directed, closely fitting in a lateral longi- 
tudinal concavity of cephalic capsule, just under the stemmata; segment I short, cylindrical, slightly emarginate ventrally, with one dorsal sensory pit; segment II subcylindrical, about twice as long as I, slightly asymmetrical (straight dorsally and distinctly convex ventrally) with three short spine-like apical setae (two dorsolateral, one ventral), and one dorsal pit; sensory appendix absent but a sensory zone visible with light microscope as a translucent ventrolateral area near apex of segment; segment III slender, subequal in length to segment II, cylindrical, with a long apical seta (antennal seta), about 3.5 times longer than the entire antenna, and three short subapical spine-like setae, one dorsal, one ventral and one lateral; one minute seta near the base of apical seta and one lateral pit. Labium with gula fused to submentum forming a gulamentum; gulamentum long, slender, weakly sclerotised, without setae; ligula absent; prementum well sclerotised, with two pairs of setae, one elongate pair dorsal and one minute pair ventral to the insertion of palpi; posterolateral margins of labium thickened; bracon present, forming a bridge in front of maxillary stipes; labial palpi inconspicuous (Figs. 2b, 2d) with segments subequal, very short, cylindrical; segment II much narrower than I, with one elongate apical sensory appendix surrounded by six-eight smaller papillae.

Thorax (Figs. 1a-1c). Segments transverse, broader than head, with terga well sclerotised; prosternum not sclerotised, sterna of meso- and metathorax slightly sclerotized, clearly defined; maximum width at mesothorax; pronotum subtrapezoidal with straight sides; meso- and metanotum subrectangular, of about equal length, with slightly curved sides; ecdysial line very thin, complete on pro-, meso- and metanotum. Pronotum two times wider than long, each half of pronotum with nine minute setae and four pits approximately placed along three transverse, subparallel rows; anterior row (AR) with two setae and two pits alternating; middle row (MR) with two small setae and one lateral pit; posterior row (PR) with five setae and one pit (from medial to lateral: one seta, one pit, four setae, lined to posterior margin); prosternum with three pairs of setae: two pairs of setae transversely lined anteriorly to procoxae, one pair between the procoxae. Mesonotum slightly shorter than pronotum, 2.5 times wider (at base) than long; AR with three minute setae (two medial, one anterior to the spiracle) and one medial pit; MR with three minute setae (one medial, one more lateral, one slightly posterior to the spiracle); PR with five minute setae and one pit (from medial to lateral: three setae, one pit slightly displaced anteriorly, two setae); three pairs of medial setae on mesosternum increasing in length from anterior to posterior, anterior pair minute and in the membranous area, second and third pairs on the sclerotised plate, posterior pair elongate. Setae of metathorax similar in number, position and relative dimensions to those of mesothorax.

Legs (Figs. 1b, 1c, 2f). Coxae short and broad, subtriangular, obliquely truncate and unsclerotised at apex, with six spine-like, subapical setae; seven minute setae and one pit basally; trochanters with medial belt of six-seven pits and three ventral setae: the medial, subapical seta on proand mesotrochanters extremely long, much longer than tibiotarsus and slightly enlarged at apex, the equivalent seta on metatrochanter much shorter than tibiotarsus and spine-like, the two apical trochanteral setae short and spine-like; basal part of trochanters without setae; femora short, modified, not swollen but distinctly enlarged from base to apex, slightly compressed laterally; pro-, meso- and metafemora about as long as relative tibiotarsus; profemur shorter than the others, meso- and metafemora similar in length; femora each with six setae and two pits, the longest ventral femoral seta (femoral seta II) much longer than femur and apically enlarged (more evidently on pro- and mesofemora), increasing in length from pro- to metafemora; femoral seta I shorter than II; tibiotarsi cylindrical, increasing in length and narrowing from pro- to metathorax, with one ventral longitudinal row of three-four spine-like setae and four-five shorter setae; claws thin and evenly curved (Fig. 2f), slightly increasing in length from pro- to metathorax, basally enlarged, with basal setae very short, near base of each claw.

Spiracles. Spiracles of mesothorax and abdominal segment I round, peritreme not protruding; mesothoracic spiracle anterolateral in position, almost double than abdominal spiracle I; abdominal spiracle I (Fig. 2g) dorsolaterally placed on laterotergites; spiracles of abdominal seg- 
ments II-VIII closed, remnants still visible at light microscope; spiracles of abdominal segment VIII (Fig. 2h) placed on projecting dorsal, coniform, horn-like evaginations of segments, with apices posteriorly directed and laterally opened.

Abdomen (Figs. 1a-1c). Subtriangular, regularly tapering from base to apex, maximum width at segment I; terga rectangular with entire posterior margins; laterotergites completely fused with medial terga (sutures still visible at light microscope) into a single tergum; terga of segments IVII with two transverse rows of setae, one anterior (AR) and one posterior (PR); median row of setae absent; each lateral half tergum I-VII with the following setation: AR with two small setae (three on tergum I) and one pit, PR with six short setae (one mediolaterally placed at level of spiracles, absent on segment I) and one pit; each half of tergum VIII with AR composed by two minute setae, PR with six setae placed as follows: two setae lateral to each conical spiracle, three setae and one pit slightly anterior and medial to the spiracle, one strong, spine-like seta, longer than the others, close and medial to the spiracle and posteriorly directed; tergum IX with AR composed of two small pairs of minute setae, PR with very short caudal setae, only slightly longer than other setae of the same tergum, and four pairs of setae and one pair of pits close to caudal pair. Sterna well sclerotised, entire, subrectangular; setation of sterna as follows: AR with one pair of minute setae and one pit, MR with one pair of short setae, PR with four pairs of spine-like, subequal setae disposed equally spaced to form a row along posterior margin of sclerites; sternum IX with three pairs of setae on PR. Abdominal apex (segment X or pygopod) membranous (Fig. 1c).

\section{Discussion}

First instar larvae of the subfamily Nemognathinae, are highly modified for phoresy on their bee hosts, except for those of the primitive tribe Stenoderini (Bologna \& Pinto 2001, Bologna et al. 2002). According to the phylogenetic studies of Bologna and Pinto (2001), among the phoretic nemognathines, the tribe Horiini characterized by nine larval synapomorphies, appeared to be the sister taxon of the tribe Nemognathini.
The latter previously was divided in two tribes, Sitarini and Nemognathini, a separation not supported by recent studies (Bologna \& Pinto 2001). Among the Nemognathini, a lineage composed of Australian taxa (probably referable to an undescribed genus: Bologna \& Pinto 2002, Bologna et al. unpublished) was basal to two complexes: one includes Zonitis, Euzonitis, Gnathium, Pszeudozonitis and an African species undetermined to genus; the second assemblage, internally unresolved, includes the sitarine genera, Nemognatha, Leptopalpus and certain species undetermined to genus.

According to the literature (Bologna \& Pinto 2001), two larval characters are partially distinctive between Euzonitis and Zonitis (a) the line of dehiscence (character number 59 in Bologna \& Pinto 2001) present on the pronotum of the first genus but incomplete or absent, respectively in the North American Z. (Neozonitis) dunniana and two Mediterranean species of Zonitis (Zonitis) ( $Z$. flava and Z. immaculata); (b) the length of metafemur vs. that of metatibiotarsus (character number 66), which is subequal in Euzonitis but shorter in the Zonitis species. Both larval features appear not definitely diagnostic between Euzonitis and Zonitis. No additional generic differences were detected by examining the same species with S.E.M. Also examined was $Z$. bellieri, a supplementary Mediterranean species probably belonging to a distinct species group of Zonitis (Bologna 1991), which has an incomplete line of dehiscence on pronotum, and the metafemur shorter than the metatibia as in the other Zonitis.

The distinction between Euzonitis and Zonitis remains scarcely supported by not clearly diagnostic larval characters and it is still based on adult features. The presence of one autopomophy in adults of Euzonitis, temporarily supports the validity of this genus, pending a revision of the Nemognathini genera of the "Zonitis lineage".

The S.E.M. study allowed a correction in the definition of some Nemognathinae larval features (here followed by the code number used by Bologna \& Pinto 2001) that were not obvious with the light microscope, which does not allow the detection of several details. (a) The medial seta of the first row on the stipes (character number 35) is short and normally pointed in both Stenodera puncticollis and S. caucasica (the latter, an addi- 


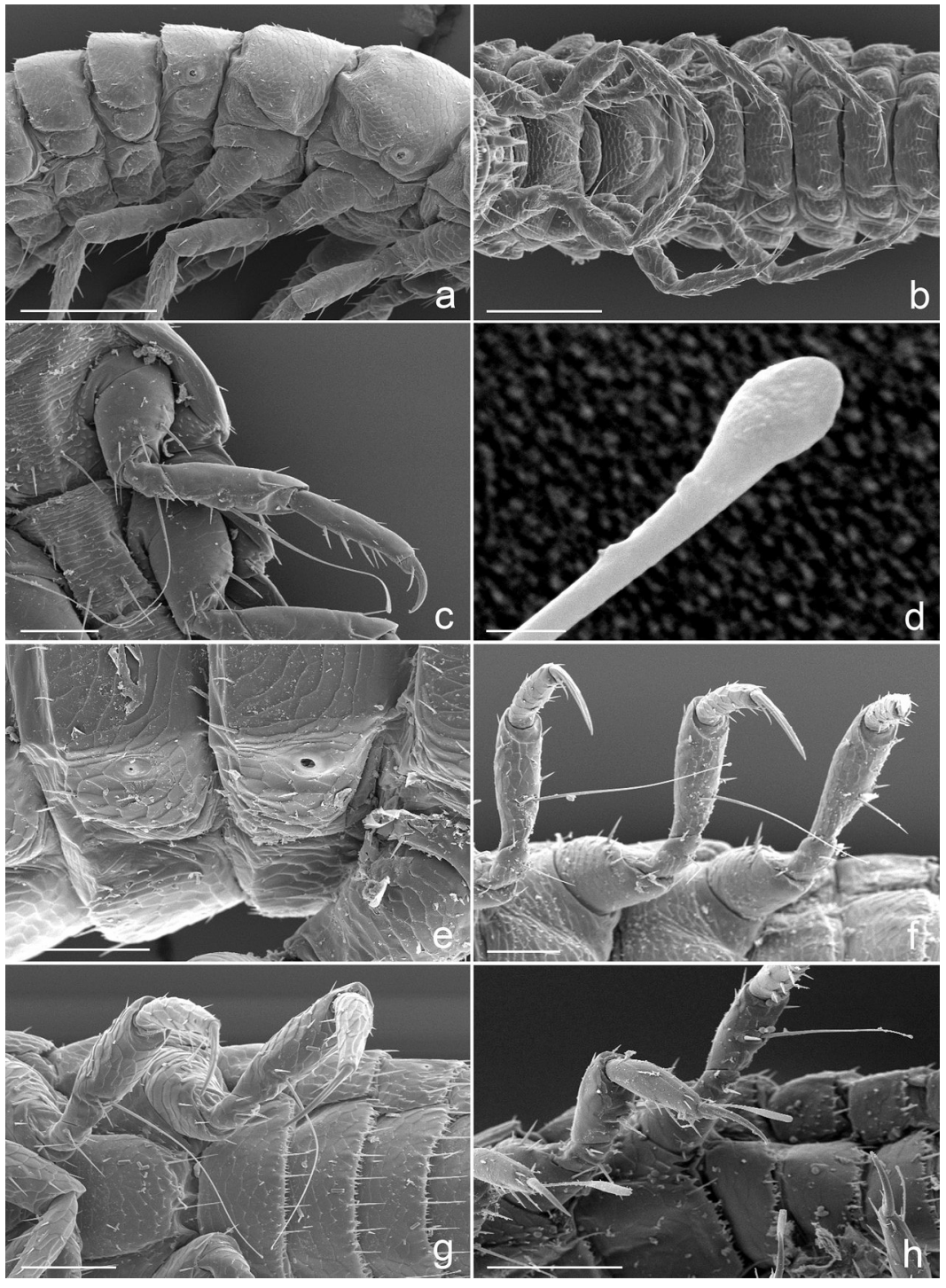

Fig. 3. First instar larva of Stenodera caucasica - a. Mesothorax, metathorax and abdominal segments I-III, right lateral view $-b$. Thorax and abdominal segments I-IV, ventral view. First instar larva of Synhoria testacea - c. Left fore leg, anterior view -d. Apex of profemoral seta. First instar larva of Nemognatha vansonie. Abdominal terga I-II, right lateral view $-f$. Left legs, anteroventral view. First instar larva of Gnathium francilloni-g. Left mid and hind legs, anteroventral view. First instar larva of Zonitis flava h. Left legs, anteroventral view. Scale bars: $100 \mu \mathrm{m}$ (Figs. ab); $50 \mu \mathrm{m}$ (Figs. c, e-h); $2 \mu \mathrm{m}$ (Fig. d). tional species recently obtained but never described), while the external seta of the second row is elongate, even if also normally pointed. On the contrary all examined Horiini and Nemognathini (Figs. 2b, 2d) have the medial seta of the first row very elongate and ampullate at the apex, a condition never observed before. (b) The subapical seta present on the ventral surface of pro- and mesotrochanters, was not previously described (Bologna \& Pinto 2001). It is elongate and apically ampullate in the examined species of Euzonitis (Fig. 1c), Zonitis bellieri (but not in Zonitis flava, see Fig. 3h), Stenoria and Synhoria (Fig. 3c), while it is short and pointed at the apex in all re- maining examined genera (Figs. 3b, $3 \mathrm{f}-3 \mathrm{~g}$ ). This similar condition in both the Zonitis-Euzonitis lineage and Stenoria analis seems due to convergence, because a similarly modified seta is present in the last mentioned genus also on the metatrochanters. (c) In the literature the three genera of the pantropical tribe Horiini, Horia, Cissites and Synhoria, were distinguished from other Nemognathinae because of the presence of one long seta with ampullate apex on pro- (Fig. 3c) and mesofemurs (seta number II; character number 67). This shape of the seta is easily visible in the Horiini and in the genus Leptopalpus with the light microscope. Actually, with the S.E.M. 
examination of other nemognathine triungulins, we detected the presence in all examined taxa of similar elongate and ampullate setae, except in Euzonitis and Zonitis, which have spatulate (Figs. $2 \mathrm{f}, 3 \mathrm{~h}$ ) instead of ampullate setae. Consequently, this condition although more evident in the Horiini, is distributed throughout the subfamily except in the primitive Stenoderini (Fig. 3b; Bologna et al. 2002). (d) A very elongate and ampullate seta, homologous to that of the pro- and mesofemur, is present also on the metafemur of Horiini and all Nemognathini, except in Nemognatha vansoni (Fig. 3f), in which probably represents a reversal. This feature was not considered by Bologna \& Pinto (2001). (e) The mesothoracic spiracle is very large only in Stenodera puncticollis, but normally shaped in S. caucasica (Fig. 3a), as in all other Nemognathinae. (f) The abdominal spiracles II-VII (character number 99) were considered as normally present in all Nemognathinae, but actually they are closed by a thin cuticular layer (Figs. 2g, 3a) except for Horiini, Gnathium and some Nemognatha (N. lurida, $N$. vansoni); moreover, these last mentioned nemognathines, have spiracles extremely reduced in size (Fig. 3e). (g) The primitive (Stenoderini, Horiini, Australian Nemognathini lineage) or derived (remaining Nemognathini) condition (Fig. 2h) of the abdominal spiracle VIII (character number 101), is confirmed by the examination of new available taxa.

Acknowledgements. We wish to thank John D. Pinto for his suggestions and all the colleagues who helped us in collecting blister beetles: Pierluigi Bombi, Enrico Calvario, Carla Marangoni, Maurizio Mei, John D. Pinto, Monica Pitzalis, Federica Turco, Marzio Zapparoli. The SEM analyses were carried out at the L.I.M.E. (Laboratorio Interdipartimentale di Microscopia Elettronica) of the University "Roma Tre", Rome.

\section{References}

Bologna, M. A. 1991: Coleoptera Meloidae. Fauna d'Italia. XXVIII. Calderini, Bologna. XIV + 544 pp.

Bologna, M. A. 2000: Biodiversity of the Meloidae (Coleoptera) of the Brandberg Massif(Namibia). - In: Kirk-
Spriggs, A. H. \& Marais, E. (eds) Dâures, Biodiversity of the Brandberg Massif, Namibia. — Cimbebasia, Mem. 9: 201-208.

Bologna, M. A. \& Di Giulio, A. 2002: Review of the Southern African genus Prolytta Kaszab, with a description of the first instar larva, and bionomic and taxonomic remarks (Coleoptera, Meloidae). - Invertebr. Syst 16: 177-194.

Bologna, M. A. \& Pinto, J. D., 2001: Phylogenetic studies of Meloidae (Coleoptera), with emphasis on the evolution of phoresy. - Syst. Entomol. 26: 33-72.

Bologna, M. A. \& Pinto, J. D., 2002: The Old World genera of Meloidae (Coleoptera): a key and synopsis. J. Nat. Hist. 36: 2013-2102.

Bologna, M. A. Di Giulio, A. \& Pinto J. D. 2002: Review of the genus Stenodera with a description of the first instar larva of S. puncticollis (Coleoptera: Meloidae). — Eur. J. Entomol. 99: 299-313.

Borchmann, F. 1942: Neue Meloiden-Arten (Col.) II. Mitt. Muench. Entomol. Ges. 32: 682-712.

Enns, W. R. 1956: A revision of the genera Nemognatha, Zonitis and Pseudozonitis (Coleoptera, Meloidae) in America North of Mexico, with a proposed new genus. - Univ. Kans. Sci. Bull. 37: 685-909.

Escherich, K. 1897: Revision der palaearktischen Zonitiden, einer Unterfamilie der Meloiden. - Verh. Nat.forsch Ver. Brünn 35: 96-132.

Kaszab, Z. 1954: Die aethiopischen Arten der Gattung Zonitis Fabr. (Coleoptera Meloidae). - Rev. Zool. Bot. Afr. 50: 17-28.

Kaszab, Z. 1969: The system of the Meloidae (Coleoptera). - Mem. Soc. Entomol. Ital. 48: 241-248.

Lawrence, J. F., 1991: Order Coleoptera. - In: Stehr, F. W. (ed.), Immature Insects vol. 2: 144-298. Kendal/Hunt, Dubuque, Iowa. 974 pp.

Lindberg, H. 1963: A contribution to the study of beetles in the Madeira islands. Results of the expeditions in 1957 and 1959. XI. Cisidae, Bostrychidae, Ptinidae, Oedemeridae, Aderidae, Meloidae. - Comm. Biol. Soc. Sci. Fen. 25: 103-104.

MacSwain, J. W. 1956: A classification of the first instar larvae of the Meloidae (Coleoptera). - Univ. Cal. Publ. Entomol. 12: i-iv, 1-182.

Parker, J. B. \& Böving, A. G. 1924: The blister beetle Tricrania sanguinipennis - biology, descriptions of different stages, and systematic relationship. — Proc. U.S. Nat. Hist. Mus. 64: 1-40, 5 pls.

Pinto, J. D. \& Bologna, M. A. 1999: The New World Genera of Meloidae (Coleoptera): a key and synopsis. - J. Nat. Hist. 33: 569-619.

Selander, R. B. 1990: Blister beetles of the genus Pseudopyrota (Coleoptera: Meloidae). — J. Kans. Entomol. Soc. 63: 46-79.

Semenov A. 1893: Meloidarum species novae. - Hor. Soc. Entomol. Ross. 27: 265-281. 